\title{
Beiträge zur Kenntnis der Nikotinsäurederivate.
}

\section{Mitteilung.}

Über Guvacin und Isoguvacin.

Von

E. Winterstein und A. Weinlagen.

(Aus dem agrikulturchemischen Laboratorium der Eidgenössischen Technischen Hochschule, Zürich.)

(Der Redaktion zugegangen am 14. August 1918.)

Der Entdecker der Arekanuß-Alkaloide, E. Jahns, ${ }^{\text {) }}$ ) hat für das Hauptalkaloid, das Arecolin, eine Formel aufgestellt, die erst viel später durch eine äußerst interessante Synthese von A. Wohl und A. Johnson ${ }^{2}$ ) bewiesen wurde. Dem Arecolin kommt hiernach die Formel I zu<smiles>COC(=O)C1CC2CCC1C2</smiles>

Für eines der Nebenalkaloide, das Jahns in nicht ganz reinem Zustand isoliert hatte, das Guvacin, wurde von dem Genannten eine Formel aufgestellt, die höchst unwahrscheinlich ist. G. Trier, ${ }^{3}$ ) welcher sich in unserem Laboratorium

1) Arch. der Pharm., Bd. 229, S. 669 (1891); Ber. d. Deutsch. Chem. Gesellsch., Bd. 21, S. 3404 (1888); ibid., Bd. 23, S. 2972 (1890); ibid., Bd. 24 ; S. 2615 (1891).

2) Ber. d. Deutsch. Chem. Gesellsch., Bd. 40, S. 4712 (1907).

$\left.{ }^{3}\right)$ Weitere Beiträge zur Kenntnis einfacher Pflanzenbasen, Diese Zeitschr., Bd. 85, S. 386 (1913); G. Trier, Über einfache Pflanzenbasen, Berlin 1912; E. Winterstein und G. Trier, Die Alkaloide, Berlin 1910, S. 293. 
mit den Nebenbasen der bezeichneten Droge beschäftigt hat, gibt dem Guvacin die durch die Formel II ausgedrückte Konstitution, wonach das Guvacin auch als

II.

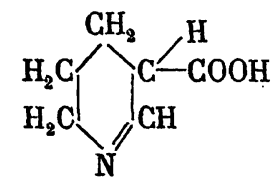

Guvacin (Trier)

ein Nikotinsäurederivat anzusehen ist. $\mathrm{Da}$ Alkaloide einer Pflanze oft enge Beziehung in ihrem Bau aufweisen, erscheint obiges Formelbild nicht unwahrscheinlich.

Nach Jahns Beobachtungen findet sich neben dem Guvacin noch ein anderes Alkaloid. Er sagt darüber folgendes: «Es mußte also in dem bei $265-270^{\circ}$ schmelzenden Basengemenge neben Guvacin noch ein anderes Alkaloid stecken, das zwar dieselbe Zusammensetzung wie dieses $z u$ besitzen scheint, sich von ihm aber durch die Fähigkeit, zwei Wasserstoffatome gegen Methyl auszutauschen, bestimmt unterscheidet».

Trier fand, daß sein Guvacin optisch aktiv ist. Die neben dem Guvacin vorkommende Base bezeichnet Trier als Isoguvacin und gibt ihm die Formel III.

III.<smiles>O=C(O)[C@H]1C[C@H]2C[C@H]1CN2</smiles>

Isoguvacin (Trier)

Im Wintersemester 1914/15 stellte uns die Firma F. Hoffmann, La-Roche \& Cie in Basel eine größere Menge konzentrierter Mutterlauge, welche bei der Darstellung des Arecolins resultiert, zur Verfügung. Wir danken auch an dieser Stelle der genannten Firma bestens.

Es gelang uns, nacb einem relativ einfachen Verfahren daraus 2 isomere Verbindungen, die wir Guvacin und Isoguvacin nennen, darzustellen. Die Ergebnisse unserer Untersuchung waren im Herbst 1916 im Manuskript fertig. Eine ausführliche Publikation erfolgt in dem Archiv für Pharmacie. ${ }^{1}$ ) Im

1) Siehe darüber die ausführliche Publikation.

Hoppe-Seyler's Zeitschrift f. physiol. Chemie. CIV. 
Hinblick auf die Veröffentlichungen von K. Freudenberg ${ }^{1}$ ) und die Arbeiten von $K$. Heß und F. Leibbrandt ${ }^{2}$ ) über den gleichen Gegenstand teilen wir an dieser Stelle die Ergebnisse unserer Untersuchungen in aller Kürze mit.

\section{I. Über das Guvacin.}

Über die Darstellung bezw. Trennung der beiden isomeren Basen ist folgendes anzugeben. Die braune, dickflüssige Mutterlauge wurde mit viel Wasser verdünnt, mit Bleiessig gereinigt; die erhaltenen Chlorhydrate wurden mit alkoholischer Sublimatlösung gefällt, aus dem Filtrat davon wurde ein schwachgefärbter Rückstand erhalten, welcher zunächst mit Alkohol behandelt und durch systematische Krystallisation in 12 Fraktionen zerlegt wurde. Die ersten acht Fraktionen schmolzen scharf bei $312^{\circ}$; die letzten drei leichter löslichen bei $231^{\circ}$. Die ersten Fraktionen gaben uns 75 g Guvacinchlorhydrat; die zweite (ca. $10 \mathrm{~g}$ ) wollen wir als Isoguvacinchlorhydrat bezeichnen.

Eigenschaften des Guvacins. Das Chlorhydrat $\mathrm{C}_{6} \mathrm{H}_{9} \mathrm{NO}_{2} \mathrm{HCl}$. Schmelzp. $312^{\circ}$; in kaltem Wasser nicht leicht löslich.

Platindoppelsalz $\left(\mathrm{C}_{6} \mathrm{H}_{9} \mathrm{NO}_{2} \mathrm{HCl}\right)_{2} \mathrm{PtCl}_{4} 4 \mathrm{H}_{2} \mathrm{O}$. Das Wasser entweicht über Calciumchlorid. Schmelzp. vom $\mathrm{H}_{2} \mathrm{O}$-freien Salz $2^{33}{ }^{\circ}$. Wenig löslich in kaltem Wasser.

Golddoppelsalz $\mathrm{C}_{6} \mathrm{H}_{9} \mathrm{NO}_{2} \mathrm{HClAuCl}_{3}$. Schmelzp. 195-197\%. Schwer löslich in kaltem Wasser.

Nitrosoguvacin. Schmelzp. $167^{\circ}$. Nadeln aus heißem Wasser. Gibt die Liebermannsche Nitrosoreaktion.

Die freie Base $\mathrm{C}_{6} \mathrm{H}_{9} \mathrm{NO}_{2}$ wurde in verschiedener Weise aus dem Chlorhydrat dargestellt. Charakteristische Prismen. Schmelzp. 293-295 ${ }^{\circ}$. Neutral gegen Lackmus. Wir fanden, daß unser Guvacin optisch inaktiv ist. Mit Eisenchlorid tritt Rotfärbung ein.

1) Ber. d. Deutsch. Ċhem. Gesellsch., Bd. 51, S. 976 (1918).

2) Ibid., Bd. 51, S. 806 (1918). Siehe auch K. Heß, ibid., Bd. 51, S. 1004 (1918). 
Das Guvacin gibt mit den meisten Alkaloidreagentien Fällungen. Mit festem Baryt erhitzt, Pyridingeruch. Bei der Reduktion mit Wasserstoff und Platin nimmt das Guvacin 2 Atome Wasserstoff auf. Das erhaltene Dihydroguvacinchlorhydrat ist in Wasser leicht, in Alkohol schwer löslich; es schmilzt bei $237^{\circ}$. Die freie Base schmilzt bei $252^{\circ}$. Das Platindoppelsalz bei $233-235^{\circ}$. Das Golddoppelsalz, prismatische Nadeln mit abgestuupften Enden, schmilzt bei 193-195 ${ }^{\circ}$. Das Quecksilberdoppelsalz ist im Alkohol nahezu unlöslich. Schmelzp. 230-231 .

Dem Dihydroguvacin muß die Formel $\mathrm{C}_{6} \mathrm{H}_{11} \mathrm{NO}_{2}$ zukommen. Eine Verbindung von dieser Zusammensetzung aber ist Hexahydronikotinsäure, welche von Ladenburg 1) eingehend untersucht worden ist. Wir haben reine Nikotinsäure ${ }^{2}$ ) mit Wasserstoff und Platinkatalyt vollständig reduziert und konnten die Angaben Ladenburgs über die Eigenschaften ihrer Derivate vollauf bestätigen. Das Dihydroguvacin und seine Derivate stimmten mit denjenigen der Hexahydronikotinsäure vollständig überein. Besonders aber ist hervorzuheben, daß auch die Schmelzpunkte der Gemische von Hexahydronikotinsäure und unserem Dihydroguvacin mit denjenigen der einzelnen Komponenten des Gemisches übereinstimmten.

Demnach muß Guvacin eine Tetrahydronikotinsäure sein. Eine $\Delta^{3}$-Tetrahydronikotinsäure, deren Chlorhydrat bei 309 bis $314^{\circ}$ schmilzt, also im Schmelzpunkt mit dem von uns dargestellten Guvacinchlorhydrat übereinstimmt, hat Wohl und Losanitsch ${ }^{3}$ ) synthetisch dargestellt. Um einen Vergleich anstellen zu können, baten wir Herrn Prof. Wohls) im Mai 1916 um gefällige Zusendung eines Präparates. Leider ging dasselbe wegen des Ausfuhrverbotes an Herrn Wohl zurück.

1) Ber. d. Deutsch. Chem. Gesellsch., Bd. 25, S. 2769 (1892).

2) Diese wurde aus Nikotin durch Oxydation mit Salpetersäure dargestellt.

3) Ber. d. Deutsch. Chem. Gesellsch., Bd. 40, S. 4701 (1907).

4) Es sei uns auch an dieser Stelle gestattet, Herrn Prof. Wo hl bestens zu danken. 
Nach den bisher erhaltenen Versuchsergebnissen ist es somit höchst wahrscheinlich, daß das Guvacin folgende Konstitution besitzen muß. Formel IV.

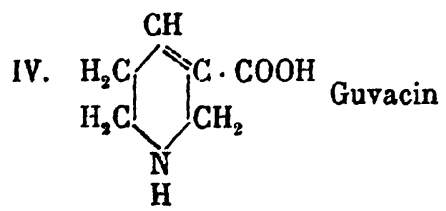

Ist unsere Auffassung richtig, so muß das Guvacin beim Methylieren ein Dimethylguvacin geben, welches mit dem von R. Willstätter ${ }^{1}$ ) dargestellten Arekaidin-Methylbetain identisch sein muß. Diese Erwartung wurde vollauf bestätigt.

$\mathrm{N}$-Dimethylguvacin wurde aus Guvacin mit Jodmethyl in methylalkoholischer Lösung dargestellt. Das Chlorhydrat dieser Base schmilzt bei 256-258 ${ }^{\circ}$. Das Platindoppelsalz krystallisiert verschieden; zuweilen Kombination von Oktaeder mit Hexaeder. Schmelzp. 253 ${ }^{\circ}$. Das Golddoppelsalz kleine glänzende, eigenartig gitterförmig übereinander gelagerte Plättchen. Schmelzp. 224-226 ${ }^{\circ}$. Das Pikrat, schwer löslich in Wasser, Plättchen. Schmelzp. 224-225 .

Das Quecksilberdoppelsalz schmilzt bei $174-176^{\circ}$. Die freie Base ist äußerst hygroskopisch. Schmelzp. $225^{\circ}$ ? Sie entfärbt Permanganatlösung. Durch Kochen mit Baryumhydroxyd tritt keine Verănderung ein. Mit den meisten Alkaloidfällungsmitteln treten Niederschläge auf.

Zum Vergleich stellten wir uns nach den Angaben von Willstätter ${ }^{1}$ ) aus Arecolin das Arekaidinbetain dar und fanden, daß die freie Base sowohl als auch ihre Derivate mit dem oben beschriebenen N-Dimethylguvacin übereinstimmten.

Das Platindoppelsalz besaß einen Gehalt von $27,19 \% \mathrm{Pt}$. Berechnet 27,11\% für $\left[\mathrm{C}_{8} \mathrm{H}_{13} \mathrm{NO}_{2} \mathrm{HCl}_{2} \mathrm{PtCl}_{4}\right.$. Demnach kommt dem Guvacin obige Formel einer $\Delta^{3}$-Tetrahydronikitinsäure zu.

\section{Das Isoguvacin.}

Das Chlorhydrat ist im Wasser leicht löslich. Es schmilzt unter Aufschäumen bei $231^{\circ}$.

1) Ber. d. Deutsch. Ghem. Gesellsch., Bd. 35, S. 615 (1902); Bd. 30, S. 729 (1897) 
Die freie Base reagiert auf Lackmus schwach sauer. Schmelzp. $220^{\circ}$.

Das Platindoppelsalz krystallisiert stets wasserfrei. Schmelzpunkt unter Zersetzung bei $235^{\circ}$.

Das Golddoppelsalz kann aus angesäuertem Wasser umkrystallisiert werden. Schmelzp. 198-200 .

Das Isoguvacin ist optisch inaktiv.

Mit den Alkaloidfällungsmitteln entstehen Niederschläge.

Beim Erhitzen einer Spur der Base mit Zinkstaub tritt intensiver Pyrrolgeruch auf, ein mit Salzsäure befeuchteter Fichtenspan wird durch die Dämpfe intensiv rot gefärbt.

Das Isoguvacin wird von Wasserstoff und Platinkatalyt langsam reduziert. Das erhaltene Chlorhydrat war nicht einheitlich.

Das Platindoppelsalz schmolz bei $225^{0} .^{1}$ )

Die aus dem Chlorhydrat mittels Phosphorwolframsäure dargestellte Base ist in Alkohol leicht löslich, während das Isoguvacin und die Hexahydronikotinsäure in Alkohol unlöslich sind.

Das Isoguvacin bildet ein Dimethylderivat, dessen Platindopnelsalz bei $252^{\circ}$ schmilzt.

$$
\left(\mathrm{C}_{8} \mathrm{H}_{13} \mathrm{NO}_{2} \mathrm{HCl}\right)_{2} \mathrm{PlCl}_{4} \text { enthält } 27,11 \% \mathrm{Pt} \text {, }
$$

$$
\text { gefunden } 26,91 \% \mathrm{Pt} \text {. }
$$

Das Isoguvacin scheint ein einfaches Pyrrolderivat zu sein, über dessen Konstitution wir nächstens berichten werden.

1) Das Golddoppelsalz des reduzierten Isoguvacins war aus verschiedenen Krystallformen zusammengesetzt, die sich völlig von dem reduzierten Guvacin, d. h. der Hexahydronikotinsäure unterschieden. 